\title{
Pediatric Oncology Patients and COVID-19: An Experience from the Tertiary COVID Care Facility in Eastern India: A Prospective Observational Study
}

\author{
Satyabrata Roy Chowdhoury1,® Himanshu Sekhar Biswas ${ }^{1} \quad$ Sumantra Raut ${ }^{1} \quad$ Subhajit Bhakta ${ }^{1, \odot}$ \\ Atanu Roy ${ }^{1, \odot}$ Swarnabindu Banerjee ${ }^{2}$ Mousumi Nandi ${ }^{1}$
}

${ }^{1}$ Department of Pediatric Medicine, Medical College and Hospital, Kolkata, West Bengal, India

${ }^{2}$ Department of Medical Oncology, Medical College and Hospital, Kolkata, West Bengal, India

\author{
Address for correspondence Subhajit Bhakta, MD, Department of \\ Pediatric Medicine, Medical College \& Hospital, 88, College Street, \\ Kolkata, West Bengal 700073, India \\ (e-mail: drsubhajitbhakta@gmail.com).
}

Ind J Med Paediatr Oncol 2021;42:130-134.

\section{Abstract}

Keywords

- COVID-19

- pediatric oncology

- pandemic

- childhood malignancy
Introduction Pediatric oncology patients presumably are one of the most vulnerable groups during this ongoing coronavirus disease 2019 (COVID-19) pandemic. Not only they are immunocompromised thanks to their inherent disease and treatment regimens, but delay in initiation and maintenance of their treatment in this pandemic era also poses great concern. But the magnitude of this effect on pediatric oncology patients has not been well established due to paucity of data.

Objective This study was proposed to assess clinicoepidemiological profile and outcome of the pediatric oncology patients who were infected with severe acute respiratory syndrome coronavirus 2 virus attending a COVID-19 care facility.

Materials and Methods This was a prospective observational study conducted in a tertiary care hospital. All confirmed oncology patients up to age 12 years who either attended the oncology outpatient department or referred to COVID-19 care center from other cancer treatment facility were included in the study from May 2020 to September 2020. Data on demography, clinical features, investigations, treatment, complications, and outcome were collected. Descriptive statistical analysis was performed and common relevant investigations were compared between non-intensive care unit (ICU) and ICU group.

Results The rate of COVID-19 positivity among the pediatric oncology patient attending oncology clinic of the hospital is $8.21 \%$. Total 28 patients ( 12 from oncology clinic of our hospital and 16 from other hospitals referred to our COVID-19 unit) were included in our study. The most common malignancy was acute lymphoblastic leukemia (64.28\%). The most common symptom was fever (64.28\%). Oxygen therapy was needed for $42.85 \%$ patients. Eight patients required ICU admission (two required invasive ventilation and one required noninvasive ventilation). Positive $\mathrm{C}$-reactive protein value was associated with severe disease requiring ICU admission. Mean delay in starting
DOI https://doi.org/

10.1055/s-0041-1732814

ISSN 0971-5851
C 2021. Indian Society of Medical and Paediatric Oncology.

This is an open access article published by Thieme under the terms of the Creative Commons Attribution-NonDerivative-NonCommercial-License, permitting copying and reproduction so long as the original work is given appropriate credit. Contents may not be used for commercial purposes, or adapted, remixed, transformed or built upon. (https://creativecommons.org/licenses/by-nc-nd/4.0/).

Thieme Medical and Scientific Publishers Private Ltd. A-12, Second Floor, Sector -2, NOIDA -201301, India 
chemotherapy in newly diagnosed cases was 28.77 days (standard deviation $=9.67$ ). One newly diagnosed patient expired due to preexisting disease.

Conclusion Though vulnerable, most of the pediatric oncology patients suffered a mild COVID-19 infection without any significant COVID-19-related morbidity and mortality. There is a significant delay in starting specific oncology therapy, that is, chemotherapy as a result of the ongoing COVID-19 pandemic in newly diagnosed pediatric oncology patients, which can increase morbidities and mortality related to malignancy.

\section{Introduction}

We are in the middle of the ongoing coronavirus disease 2019 "COVID-19" pandemic that has emerged from Wuhan, China, and till date has spread over 220 countries and territories across the continents. It has affected over 50 million people worldwide and $\sim 1.3$ million people have died as of mid-November 2020; India has over 9 million cases with over 1.3 lakhs case fatalities. ${ }^{1}$ The data so far revealed that pediatric population constitutes a less percentage of total cases than adults and so far favorably having a mostly milder form of the disease. ${ }^{2}$ The most vulnerable population is the elderly population with significant comorbidities. In children, presumably the high-risk cohorts are also those with other comorbidities and immunocompromised patients. ${ }^{3,4}$ Malignancy is definitely a significant comorbid condition and an inherent immune-deficient state, compounded by the use of chemotherapeutic agents. The pathogenesis and manifestations of COVID-19 are predominantly determined by the fine interplay between proinflammatory and anti-inflammatory mechanisms inherent to our immune system. As pediatric population constitutes a smaller number of cases compared with adults, there is paucity of data on pediatric population from various parts of the world. The pediatric scenario of the disease is still emerging in respect to disease understanding and devising a standard treatment protocol. The effect of chemotherapy and radiotherapy on the risk of progression of an asymptomatic or mildly symptomatic severe acute respiratory syndrome coronavirus 2 (SARS-CoV-2) infection toward a severe or lethal form of COVID-19 is not really known, ${ }^{5}$ nor do we know the clear guidelines whether to continue the ongoing therapeutic protocol in a symptomatic COVID-19 positive patient. These precautions are in sync with the data from adult oncology patients showing that recent chemotherapy or oncology surgery was a risk factor for a more severe COVID-19 infection. ${ }^{6}$ We intend to assess clinicoepidemiological profile and outcome of the pediatric oncology patients who got infected with COVID-19 virus.

\section{Materials and Methods}

This was a prospective observational study conducted in a tertiary care hospital in eastern India, which is an oncology center, as well as a dedicated tertiary COVID care center for pediatric patients since the start of the pandemic.

The pediatric oncological patients who attended to the oncology outpatient department from May 2020 to September 2020 were subjected to COVID-19 real-time polymerase chain reaction (RT-PCR) test. Those patients who tested positive were included in the study along with COVID-19 positive oncological patients who were referred to our COVID-19 referral center from various parts of the region. The estimated sample size was calculated to be 27 with a COVID-19 positivity rate of $11.2 \%$ among pediatric oncology patients and an allowable error rate of $12 \%$.

The positive patients were referred to pediatric COVID-19 care facility for further treatment. Demographic, clinical, investigation, treatment, complications, and outcome-related data were collected from all pediatric oncology patients who were COVID-19 RT-PCR positive either from our hospital or from other hospital referred to our facility. Basic investigations like complete hemogram, liver function tests, renal function tests, electrolytes, blood and urine cultures, inflammatory markers like C-reactive protein (CRP), ferritin, procalcitonin, and D-dimer were assessed as required by the clinician. Severity of COVID infection was categorized based on Guidelines issued by Ministry of Health and Family Welfare (MOHFW), Govt. of India. ${ }^{8}$ The primary outcome was measured as clinical and laboratory profile of the study population. Delay in initiation of chemotherapy, average time to become RT-PCR negative, mortality, and laboratory parameters associated with pediatric intensive care unit admission were considered as secondary outcomes.

\section{Statistical Analysis}

Patient data were recorded in predesigned case record form and transferred to an excel sheet. Descriptive statistical analysis performed with STATA 13 software (StataCorp. 2013. Stata Statistical Software: Release 13. College Station, Texas, United States: StataCorp LP) for Windows with Microsoft Excel and Microsoft Word was used to prepare tables. Results on continuous measurements are presented as mean \pm SD (standard deviation) for parametric data and median (interquartile range) for nonparametric quantitative variables. Results on categorical measurements are presented in number (\%). Wilcoxon rank-sum test was used to compare the median of nonparametric variables between non-intensive care unit 
(ICU) and ICU groups of patients. Chi-squared/Fisher's exact test was used to find the significance of study parameters on categorical scale between two groups.

\section{Ethics}

This study was approved by the Institutional Ethics Committee (Board Name: Institutional Ethical Committee, Medical College, Kolkata, Reg. No - ECR/287/Inst/WB/2013/ RR19, Ref no: MC/KOL/IEC/NON-SPON/775/09/20 dated September 3, 2020). The procedures followed were in accordance with the ethical standards of the responsible committee on human experimentation and with the Helsinki Declaration of 1964, as revised in 2013. Informed patient consent was obtained from all the patients prior to enrollment in the study.

\section{Results}

During the study period, a total of 146 pediatric oncology patients attended our hospital and they were assessed with RT-PCR for SARS-CoV-2. Of 146 patients, only 12 (8.21\%) patients tested positive and were admitted in the COVID-19 ward. A total of 16 patients were referred from various parts of the state to our COVID-19 referral center. So, total positive oncological cases were 28 who were treated in our center during the study period. The mean age group of the 28 positive patients is 5.52 (minimum 1 year and maximum 11 years). Male sex constitutes 13 (46.42\%) and female sex constitutes 15 (53.57\%). Out of 28 positive oncological patients, eighteen (64.28\%) patients had acute lymphoblastic leukemia and four patients (14.28\%) had acute myeloid leukemia. Other malignancies found in our study population include chronic myeloid leukemia, retinoblastoma, Wilms tumor, Burkitt lymphoma, and central nervous system tumors ( - Table $\mathbf{1}$ ). Nine patients were newly diagnosed with malignancy and were yet to start chemotherapy.

Of total 28 patients, eight patients had severe disease requiring ICU admission and the remaining 20 patients had nonsevere disease course and were managed in general pediatric ward. Fever was the most common symptom (64.28\%) followed by cough (42.85\%). Six (21.85\%) patients had breathing difficulty (-Table 2). Oxygen therapy was needed in 12 patients (42.85\%) out of total 28 patients at some point of time. All patients who only needed oxygen were given via nonrebreathing mask. Other respiratory supports like high flow nasal cannula were needed in three patients, one

Table 1 Distribution of oncological problems

\begin{tabular}{|l|l|}
\hline Oncological problems & $N=28 ;[n(\%)]$ \\
\hline Acute lymphoblastic leukemia & $18(64.28 \%)$ \\
\hline Acute myeloid leukemia & $4(14.28 \%)$ \\
\hline Chronic myeloid leukemia & $1(3.57 \%)$ \\
\hline Retinoblastoma & $1(3.57 \%)$ \\
\hline Wilms tumor & $2(7.17 \%)$ \\
\hline Cerebellar space-occupying lesion & $1(3.57 \%)$ \\
\hline Burkitt lymphoma & $1(3.57 \%)$ \\
\hline
\end{tabular}

Table 2 Presenting symptoms among symptomatic patients

\begin{tabular}{|l|l|}
\hline Presenting symptoms & $N=28 ;[n(\%)]$ \\
\hline Fever & $18(64.28 \%)$ \\
\hline Cough & $12(42.85 \%)$ \\
\hline Respiratory distress & $6(21.42 \%)$ \\
\hline Bleeding manifestation & $5(17.85 \%)$ \\
\hline Raised intracranial tension & $3(10.17 \%)$ \\
\hline Shock/hemodynamic instability & $3(10.17 \%)$ \\
\hline Acute respiratory distress syndrome & $3(10.17 \%)$ \\
\hline Superior vena cava syndrome & $1(3.57 \%)$ \\
\hline
\end{tabular}

Table 3 Respiratory support and specific therapy

\begin{tabular}{|l|l|}
\hline Method & $N=28 ;[n(\%)]$ \\
\hline Only oxygen therapy & $12(42.85 \%)$ \\
\hline High-flow nasal cannula & $3(10.17 \%)$ \\
\hline Noninvasive ventilation & $1(3.57 \%)$ \\
\hline Invasive ventilation & $2(7.17 \%)$ \\
\hline Tab hydroxychloroquine & $2(7.17 \%)$ \\
\hline Inj. remdesivir & $2(7.17 \%)$ \\
\hline Tab azithromycin & $10(35.71 \%)$ \\
\hline
\end{tabular}

patient required noninvasive ventilation, and two patients required invasive ventilation. Specific therapy like hydroxychloroquine was started in two patients and two were given remdesivir injections. Oral azithromycin was given in 10 patients. All nonsevere patients were managed symptomatically and treated conservatively with vitamin $\mathrm{C}$ and zinc supplementation ( $\mathbf{- T a b l e} \mathbf{3}$ ).

One patient died due to preexisting disease. The cause of death was intracerebral hemorrhage with raised intracranial tension with central diabetes insipidus in a patient of acute myeloid leukemia with hyperleukocytosis. Remaining 27 patients were successfully discharged after testing RT-PCR negative for COVID-19 and were further treated at oncomedicine facilities. The mean hospital stay of the 28 positive oncological patients was 14.85 days and the mean duration to become swab negative was 12.70 days $(\mathrm{SD}=3.798)$.

Though average hemoglobin, absolute neutrophil count (ANC) and platelet count were low and ferritin level was high among the severe group it was not statistically significant. Association between positive CRP test and severe disease course requiring ICU admission was found to be reaching near significance $(p=0.055)$ ( - Table 4$)$.

Due to this coronavirus pandemic mean delay among the newly diagnosed patients $(n=9)$ to start specific therapy for cancer was 28.77 days $(S D=9.67)$ and those who were already under therapy $(n=19)$ had mean delay in receiving next scheduled chemotherapy was 14.38 days $(S D=10.07)$.

\section{Discussion}

Pediatric patients are being less affected by the COVID-19 infection during this pandemic. But recent evidence suggests that younger children ( $<5$ years) can develop severe disease 
Table 4 Comparison of laboratory parameters between non-ICU and ICU group

\begin{tabular}{|l|l|l|l|l|}
\hline Laboratory parameters & Non-ICU $(\boldsymbol{n}=\mathbf{2 0 , 7 1 . 4 3 \% )}$ & ICU $(\boldsymbol{n}=\mathbf{8 , 2 8 . 5 7 \% )}$ & $\boldsymbol{p}$-Value & Interpretation \\
\hline $\begin{array}{l}\text { Hemoglobin (median [IQR1, } \\
\text { IQR2]) }\end{array}$ & $9(7.25,10.6)$ & $8(7.25,8.75)$ & 0.344 & Nonsignificant \\
\hline ANC (median [IQR1, IQR2]) & $1,278(750,1,838)$ & $690(275,1,710)$ & 0.263 & Nonsignificant \\
\hline $\begin{array}{l}\text { Platelet count (median [IQR1, } \\
\text { IQR2]) }\end{array}$ & $77,000(33,000,2,10,000)$ & $53,000(21,000,85,000)$ & 0.308 & Nonsignificant \\
\hline $\begin{array}{l}\text { Ferritin (median [IQR1, } \\
\text { IQR2]) }\end{array}$ & $220(1,10,505)$ & $420(250,950)$ & 0.114 & Nonsignificant \\
\hline CRP (mg/dl) & 35 & 75 & 0.055 & Nearly significant \\
\hline
\end{tabular}

Abbreviations: ANC, absolute neutrophil count; CRP, C-reactive protein; ICU, intensive care unit; IQR, interquartile range.

course. ${ }^{9}$ Those who suffered from severe disease usually have comorbidities and one of the most important comorbidity in this age group is childhood malignancy. Zhang et al (28 cases) and Zhang et al (67 cases) reported a higher prevalence of cancer patients with COVID-19 infections compared with the overall population (1 vs. $0.29 \%$ ). ${ }^{11}$ It is well known that other viruses, including human corona viruses, have a more severe disease course in immunocompromised children. ${ }^{12}$ Since pediatric malignant tumors are themselves a potential immunosuppressed state and their treatment modalities also suppress immunity, COVID-19 infection is expected to lead to a more serious illness. No robust data exist to date regarding the infection incidence and prevalence in pediatric cancer patients. In our study, we found that only $8.21 \%$ of pediatric oncology patients visiting oncology day care got infected. In a study conducted by Boulad et al in United States found that out of 178 pediatric oncology patients screened for COVID-19 infection only 20 tested positive (11.2\%). ${ }^{7}$

Among the total 28 pediatric cancer patients treated in our pediatric COVID-19 facility, only 8 needed ICU admissions and one succumbed due to preexisting disease. About $42.85 \%$ required oxygen therapy and $10.17 \%$ patients were on high-flow nasal oxygen. One patient required noninvasive and other two patients required invasive ventilation. Reports from other countries also have shown fewer cases and a less severe course of disease among the pediatric oncology patients against our expectation. Five pediatric cancer cases have been reported from Italy and all of them had a self-limiting course; only two patients required hospitalization. ${ }^{13}$ However, French oncology center reported 33 confirmed cases of COVID-19 among which only five required ICU treatment and no death was reported. ${ }^{14}$ Another six cases have been reported from the United States in which all of them had mild symptoms. ${ }^{15}$

In another report from Madrid, 15 pediatric patients were found as COVID-19 positive with malignancy and among them seven patients required hospitalization. The most common symptom was fever (67\%) followed by cough (40\%). ${ }^{16}$ In our study, we also found that fever is the most common symptom (64.28\%), followed by cough (42.85\%). Other more severe symptoms like respiratory distress (21.42\%), shock (10.17\%), and acute respiratory distress syndrome (10.17\%) were fewer in number.

In a multicenter survey among pediatric oncology centers, only nine out of 200 symptomatic or suspected patients tested positive for SARS-CoV-2. None of them required intensive care or ventilator support, and only two patients were treated with hydroxychloroquine together with lopinavir/ritonavir in one patient. ${ }^{17}$ In our study, most of the patients were treated conservatively. But two patients were treated with hydroxychloroquine and two patients received remdesivir.

We did not find any significant difference between hemoglobin level, ANC, platelet count, or ferritin level among the non-ICU and ICU group. But CRP level was near-significantly positive among the ICU group. But as the study population is very small, it is very difficult to comment on the clinical significance of this finding.

In our study, we also found that during pandemic mean delay among the newly diagnosed patients to start specific therapy for cancer was 28.77 days $(S D=9.67)$ and those who were already under therapy had mean delay in receiving next scheduled chemotherapy was 14.38 days (SD $=10.07)$. This is very significant and may be responsible for more oncology-related complications and one death among our study population. A survey conducted in France showed degradation of medical care and supportive care in pediatric oncology due to ongoing COVID-19 pandemic. ${ }^{18} \mathrm{~A}$ small case series from Italy has described delayed presentation of pediatric patients to emergency during a week at the height of COVID-19 pandemic. Three of these patients were newly diagnosed pediatric malignancy cases and one of them expired..$^{19}$ In a country like India, where health care facility is not so easily accessible, COVID-19 pandemic will have a more profound effect on pediatric oncology patients who require more regular and frequent follow-ups and interventions. Our study has reflected a similar picture.

The most important limitation of our study is a small sample size and the fact that we have not done a follow-up study to assess whether this interruption or delay in chemotherapy causes any significant effect on primary disease. We, therefore, propose a multicenter study with a larger sample size and follow-up data. 


\section{Conclusion}

The severity and overall outcome in COVID-positive pediatric oncology patients are not different from the general pediatric population. However, there is an unwarranted interruption or delay in chemotherapy due to the ongoing pandemic. It is very important for health care professionals and health care providers to ensure that pediatric cancer patients get an uninterrupted treatment facility even during a pandemic.

\section{Funding}

The research was not funded by any organizations.

\section{Authors' Contribution}

Satyabrata Roy Chowdhury, Subhajit Bhakta, and Swarnabindu Banerjee conceptualized, designed, and defined the intellectual content. Himangshu Sekhar Biswas acquired data and did research for the literature. Sumantra Raut and Satyabrata Roy Chowdhury did data and statistical analysis. Subhajit Bhakta prepared the manuscript. Atanu Roy and Mousumi Nandi edited and reviewed the manuscript. The corresponding author takes responsibility for integrity of the work.

\section{Conflict of Interest}

None.

\section{References}

1 Dong E, Du H, Gardner L. An interactive web-based dashboard to track COVID-19 in real time. Lancet Infect Dis 2020;20(5):53353410.1016/S1473-3099(20)30120-1Erratum in: Lancet Infect Dis. 2020 Sep;20(9):e215

2 Shekerdemian LS, Mahmood NR, Wolfe KK, et al. International COVID-19 PICU Collaborative. Characteristics and outcomes of children with coronavirus disease 2019 (COVID-19) infection admitted to US and Canadian pediatric intensive care units. JAMA Pediatr 2020;174(9):868-873

3 Guan WJ, Liang WH, Zhao Y, et al. China Medical Treatment Expert Group for COVID-19. Comorbidity and its impact on 1590 patients with COVID-19 in China: a nationwide analysis. Eur Respir J 2020;55(5):200054710.1183/13993003.00547-2020

4 Gao Y, Chen Y, Liu M, Shi S, Tian J. Impacts of immunosuppression and immunodeficiency on COVID-19: a systematic review and meta-analysis. J Infect 2020;81(2):e93-e95

5 Cesaro S, Compagno F, Zama D, et al. Screening for SARS-CoV-2 infection in pediatric oncology patients during the epidemic peak in Italy. Pediatr Blood Cancer 2020;67(8):e2846610.1002/pbc.28466
6 Liang W, Guan W, Chen R, et al. Cancer patients in SARS-CoV-2 infection: a nationwide analysis in China. Lancet Oncol 2020;21(3):335-337

7 Boulad F, Kamboj M, Bouvier N, Mauguen A, Kung AL. COVID-19 in children with cancer in New York City. JAMA Oncol 2020;6(9):1459-1460

8 MOHFW govt of India. Clinical Management Protocol:Covid19. India:2020 June13.Availablefrom: https://www.mohfw.gov. in/pdf/ClinicalManagementProtocolforCOVID19.pdf. Accessed June 30, 2021

9 Dong Y, Mo X, Hu Y, et al. Epidemiology of COVID-19 among children in China. Pediatrics 2020;145(6):e2020070210.1542/ peds.2020-0702

10 Jain V, Yuan JM. Predictive symptoms and comorbidities for severe COVID-19 and intensive care unit admission: a systematic review and meta-analysis. Int J Public Health 2020;65(5):533-546

11 Zhang L, Zhu F, Xie L, et al. Clinical characteristics of COVID-19-infected cancer patients: a retrospective case study in three hospitals within Wuhan, China. Ann Oncol 2020;31(7):894-901

12 Ogimi C, Englund JA, Bradford MC, Qin X, Boeckh M, Waghmare A. Characteristics and outcomes of coronavirus infection in children: the role of viral factors and an immunocompromised state. J Pediatric Infect Dis Soc 2019;8(1):21-28

13 Balduzzi A, Brivio E, Rovelli A, et al. Lessons after the early management of the COVID-19 outbreak in a pediatric transplant and hemato-oncology center embedded within a COVID-19 dedicated hospital in Lombardia, Italy. Estote parati. Bone Marrow Transplant 2020;55(10):1900-1905

14 André N, Rouger-Gaudichon J, Brethon B, et al. COVID-19 in pediatric oncology from French pediatric oncology and hematology centers: high risk of severe forms? Pediatr Blood Cancer 2020;67(7):e2839210.1002/pbc.28392

15 Rossoff J, Patel AB, Muscat E, Kociolek LK, Muller WJ. Benign course of SARS-CoV-2 infection in a series of pediatric oncology patients. Pediatr Blood Cancer 2020;67(9):e2850410.1002/ pbc. 28504

16 de Rojas T, Pérez-Martínez A, Cela E, et al. COVID-19 infection in children and adolescents with cancer in Madrid. Pediatr Blood Cancer 2020;67(7):e2839710.1002/pbc.28397

17 Hrusak O, Kalina T, Wolf J, et al. Flash survey on severe acute respiratory syndrome coronavirus- 2 infections in paediatric patients on anticancer treatment. Eur J Cancer 2020;132:11-16

18 Rouger-Gaudichon J, Gariazzo L, Thébault E, et al. Impact of COVID-19 on cancer care: a survey from the French Society of Pediatric Oncology (SFCE. Pediatr Blood Cancer 2021;68(1):e2855410.1002/pbc.28554

19 Lazzerini M, Barbi E, Apicella A, Marchetti F, Cardinale F, Trobia G. Delayed access or provision of care in Italy resulting from fear of COVID-19. Lancet Child Adolesc Health 2020;4(5):e10-e11 